IJMMS 30:6 (2002) 327-338

PII. S0161171202004167

http://ijmms.hindawi.com

(c) Hindawi Publishing Corp.

\title{
INITIAL-BOUNDARY VALUE PROBLEM WITH A NONLOCAL CONDITION FOR A VISCOSITY EQUATION
}

\author{
ABDELFATAH BOUZIANI
}

Received 9 October 1999

\begin{abstract}
This paper deals with the proof of the existence, uniqueness, and continuous dependence of a strong solution upon the data, for an initial-boundary value problem which combine Neumann and integral conditions for a viscosity equation. The proof is based on an energy inequality and on the density of the range of the linear operator corresponding to the abstract formulation of the studied problem.
\end{abstract}

2000 Mathematics Subject Classification: 35L20, 35L82, 35B45, 35D05, 35B30.

1. Formulation of the problem. In this paper, we deal with a class of hyperbolic equations with time- and space-variable characteristics, with a nonlocal boundary condition. The precise statement of the problem is as follows: let $\beta>0, T>0$, and $Q=\left\{(x, t) \in \mathbb{R}^{2}: \alpha<x<\beta, 0<t<T\right\}$. Find a function $v(x, t),(x, t) \in \bar{Q}$, satisfying

$$
\mathscr{L} v=\frac{\partial^{2} v}{\partial t^{2}}-\frac{\partial}{\partial x}\left(a(x, t) \frac{\partial v}{\partial x}\right)-\frac{\partial^{2}}{\partial t \partial x}\left(b(x, t) \frac{\partial v}{\partial x}\right)+c(x, t) v=\mathrm{f}(x, t),
$$

the initial condition

$$
\begin{aligned}
& \ell_{1} v=v(x, 0)=\Phi(x), \quad x \in(\alpha, \beta), \\
& \ell_{2} v=\frac{\partial v(x, 0)}{\partial t}=\Psi(x), \quad x \in(\alpha, \beta),
\end{aligned}
$$

the Neumann condition

$$
\frac{\partial v(\alpha, t)}{\partial x}=\mu(t), \quad t \in(0, T)
$$

and the integral condition

$$
\int_{\alpha}^{\beta} v(x, t) d x=E(t), \quad t \in(0, T),
$$

where $\Phi, \Psi, \mu, E, a, b, c$, and $\mathrm{f}$ are known functions. 
AsSUMPTION 1.1. For all $(x, t) \in \bar{Q}$, we assume that

$$
\begin{aligned}
& c_{0} \leq a(x, t) \leq c_{1}, \quad \frac{\partial a(x, t)}{\partial t} \leq c_{2}, \quad \frac{\partial a(x, t)}{\partial x} \leq c_{3}, \\
& c_{4} \leq b(x, t), \quad c_{5} \leq \frac{\partial b(x, t)}{\partial t} \leq c_{6}, \quad \frac{\partial b(x, t)}{\partial x} \leq c_{7}, \\
& \frac{\partial^{2} b(x, t)}{\partial t^{2}} \leq c_{8}, \quad \frac{\partial^{2} b(x, t)}{\partial x \partial t} \leq c_{9}, \quad c(x, t) \leq c_{10} .
\end{aligned}
$$

AsSUMPTION 1.2. For all $(x, t) \in \bar{Q}$, we assume that

$$
\frac{\partial^{2} a(x, t)}{\partial x \partial t} \leq c_{11}, \quad \frac{\partial^{2} b(x, t)}{\partial x^{2}} \leq c_{12}, \quad \frac{\partial^{3} b(x, t)}{\partial x \partial t^{2}} \leq c_{13}
$$

In Assumptions 1.1, 1.2, and in the rest of the paper, we assume that $c_{i}$, where $i=0, \ldots, 17$, are positive constants.

The data satisfies the following compatibility conditions:

$$
\begin{array}{ll}
\frac{d \Phi(\alpha)}{d x}=\mu(0), & \int_{\alpha}^{\beta} \Phi(x) d x=E(0), \\
\frac{d \Psi(\alpha)}{d x}=\mu^{\prime}(0), & \int_{\alpha}^{\beta} \Psi(x) d x=E^{\prime}(0) .
\end{array}
$$

Several authors investigated the initial-boundary value problems in one space variable, which involve an integral over the spatial domain of a function of the desired solution that may appear in a boundary condition. Along a different line, problems for parabolic equations which combine classical and integral conditions were considered by Batten [1], Ionkin [12], Cannon et al. [8, 9, 10, 11], Yurchuk [16], Lin [13], BenouarYurchuk [2], Shi [15], Bouziani et al. [7, 14]. However, most of these papers considered particular situations like heat equation in the rectangle $(0,1) \times(0, T)$. Problems with only boundary integral conditions for a second-order parabolic equation have been treated in Bouziani-Benouar [5], and for a 2m-parabolic equation in Bouziani [4]. Recently, a problem of this type for second-order pluriparabolic equation is studied in Bouziani [6].

In this paper, the existence, uniqueness, and continuous dependence of a strong solution upon the data of problem (1.1), (1.2), (1.3), and (1.4) are demonstrated. We use a functional analysis method based on an energy inequality and on the density of the range of the linear operator corresponding to the abstract formulation of the considered problem.

To this end, we reduce the inhomogeneous boundary conditions (1.3) and (1.4) to homogeneous conditions, by introducing a new unknown function $u$ defined by $u(x, t)=v(x, t)+K(x, t)$, where

$$
K(x, t)=\frac{(x-\alpha)}{2(\alpha-\beta)}\left\{(3 x-\alpha-2 \beta) \mu(t)-\frac{6(x-\alpha)}{(\beta-\alpha)^{2}} E(t)\right\}
$$


Then, problem (1.1), (1.2), (1.3), and (1.4) becomes

$$
\begin{gathered}
\mathscr{L} u=\mathrm{f}(x, t)+\mathscr{L} K(x, t)=f(x, t), \\
\ell_{1} u=u(x, 0)=\Phi(x)+\ell_{1} K=\varphi(x), \\
\ell_{2} u=\frac{\partial u(x, 0)}{\partial t}=\Psi(x)+\ell_{2} K=x(x), \\
\frac{\partial u(\alpha, t)}{\partial x}=0, \\
\int_{\alpha}^{\beta} u(x, t) d x=0 .
\end{gathered}
$$

Here we assume that the functions $\varphi$ and $\varkappa$ satisfy conditions of the form (1.11) and (1.12), that is,

$$
\frac{d \varphi(\alpha)}{d x}=0, \quad \int_{\alpha}^{\beta} \varphi(x) d x=0, \quad \frac{d \varkappa(\alpha)}{d x}=0, \quad \int_{\alpha}^{\beta} \varkappa(x) d x=0 .
$$

Instead of searching for the function $v$, we search for the function $u$. So the solution of problem (1.9), (1.10), (1.11), and (1.12) will be given by $v(x, t)=u(x, t)-K(x, t)$.

2. Energy inequality and its consequences. The solution of problem (1.9), (1.10), (1.11), and (1.12) can be considered as a solution of the operator equation

$$
L u=(f, \varphi, \varkappa)
$$

where $L=\left(\mathscr{L}, \ell_{1}, \ell_{2}\right)$. The operator $L$ maps from $B$ to $F$, where $B$ is the Banach space consisting of functions $\mathfrak{I}_{x} u \in L^{2}(Q)$, where $\mathfrak{J}_{x} u=\int_{\alpha}^{x} u(\xi, \cdot) d \xi$, having finite norm:

$$
\|u\|_{B}=\left\{\left\|\frac{\partial u}{\partial t}\right\|_{L^{2}(Q)}^{2}+\|u\|_{C\left(0, T ; L^{2}(\alpha, \beta)\right)}^{2}+\left\|\mathfrak{J}_{x} \frac{\partial u}{\partial t}\right\|_{C\left(0, T ; L^{2}(\alpha, \beta)\right)}^{2}\right\}^{1 / 2},
$$

and $F$ is the Hilbert space with the finite norm

$$
\|L u\|_{F}=\left\{\left\|\mathfrak{J}_{x} \mathscr{L} u\right\|_{L^{2}(Q)}^{2}+\left\|\ell_{1} u\right\|_{L^{2}(\alpha, \beta)}^{2}+\left\|\mathfrak{J}_{x} \ell_{2} u\right\|_{L^{2}(\alpha, \beta)}^{2}\right\}^{1 / 2} .
$$

The domain $D(L)$ of the operator $L$ is the set of all functions $u$ such that $\mathfrak{J}_{x} u \in$ $L^{2}(Q)$ for which $\mathfrak{I}_{x}(\partial u / \partial t), \mathfrak{I}_{x}\left(\partial^{2} u / \partial t^{2}\right), \mathfrak{I}_{x}\left(\partial^{2} u / \partial x^{2}\right) \in L^{2}(Q)$, and satisfying (1.11) and (1.12)

THEOREM 2.1. Let Assumption 1.1 be fulfilled. Then the a priori estimate

$$
\|u\|_{B} \leq C\|L u\|_{F}
$$

holds for any function $u \in D(L)$, where $C$ is a positive constant independent of $u$. 
Proof. Applying operator $\mathfrak{J}_{x}$ to (1.9) by taking into account condition (1.11), multiplying the obtained equality with $2 \mathfrak{J}_{x}(\partial u / \partial t)$, and integrating over $Q^{\top}:=(\alpha, \beta) \times$ $(0, \tau)$, where $0 \leq \tau \leq T$. Observe that

$$
\begin{aligned}
2 \int_{Q^{\top}} & \mathfrak{I}_{x} \frac{\partial^{2} u}{\partial t^{2}} \mathfrak{J}_{x} \frac{\partial u}{\partial t} d x d t-2 \int_{Q^{\top}} a(x, t) \frac{\partial u}{\partial x} \mathfrak{I}_{x} \frac{\partial u}{\partial t} d x d t \\
& -2 \int_{Q^{\top}} \frac{\partial}{\partial t}\left(b(x, t) \frac{\partial u}{\partial x}\right) \mathfrak{I}_{x} \frac{\partial u}{\partial t} d x d t \\
& +2 \int_{Q^{\top}} \mathfrak{J}_{x}(c(\xi, t) u) \mathfrak{I}_{x} \frac{\partial u}{\partial t} d x d t \\
= & 2 \int_{Q^{\tau}} \mathfrak{I}_{x} f \mathfrak{J}_{x} \frac{\partial u}{\partial t} d x d t .
\end{aligned}
$$

Integrating by parts the first three integrals on the left-hand side of (2.5), we obtain

$$
\begin{aligned}
& 2 \int_{Q^{\tau}} \mathfrak{I}_{x} \frac{\partial^{2} u}{\partial t^{2}} \mathfrak{I}_{x} \frac{\partial u}{\partial t} d x d t=\int_{\alpha}^{\beta}\left(\mathfrak{J}_{x} \frac{\partial u(\xi, \tau)}{\partial t}\right)^{2} d x-\int_{\alpha}^{\beta}\left(\mathfrak{I}_{x} \mathcal{x}\right)^{2} d x \\
& -2 \int_{Q^{\tau}} a \frac{\partial u}{\partial x} \mathfrak{J}_{x} \frac{\partial u}{\partial t} d x d t=\int_{\alpha}^{\beta} a(x, \tau) u^{2}(x, \tau) d x-\int_{\alpha}^{\beta} a(x, 0) \varphi^{2} d x \\
& -\int_{Q^{\tau}} \frac{\partial a}{\partial t} u^{2} d x d t+2 \int_{Q^{\tau}} \frac{\partial a}{\partial x} u \mathfrak{J}_{x} \frac{\partial u}{\partial t} d x d t \\
& -2 \int_{Q^{\tau}} \frac{\partial}{\partial t}\left(b \frac{\partial u}{\partial x}\right) \mathfrak{I}_{x} \frac{\partial u}{\partial t} d x d t=2 \int_{Q^{\tau}} b\left(\frac{\partial u}{\partial t}\right)^{2} d x d t+\int_{\alpha}^{\beta} \frac{\partial b(x, \tau)}{\partial t} u^{2}(x, \tau) d x \\
& -\int_{\alpha}^{\beta} \frac{\partial b(x, 0)}{\partial t} \varphi^{2} d x-\int_{Q^{\top}} \frac{\partial^{2} b}{\partial t^{2}} u^{2} d x d t \\
& +2 \int_{Q^{\tau}} \frac{\partial b}{\partial x} \frac{\partial u}{\partial t} \mathfrak{J}_{x} \frac{\partial u}{\partial t} d x d t+2 \int_{Q^{\tau}} \frac{\partial^{2} b}{\partial x \partial t} u \mathfrak{J}_{x} \frac{\partial u}{\partial t} d x d t .
\end{aligned}
$$

Substituting (2.6) into (2.5), we get

$$
\begin{aligned}
2 \int_{Q^{\tau}} & b\left(\frac{\partial u}{\partial t}\right)^{2} d x d t+\int_{\alpha}^{\beta}\left\{\left(a+\frac{\partial b}{\partial t}\right) u^{2}(x, \tau)+\left(\mathfrak{I}_{x} \frac{\partial u(\xi, \tau)}{\partial t}\right)^{2}\right\} d x \\
= & 2 \int_{Q^{\tau}} \mathfrak{I}_{x} f \mathfrak{I}_{x} \frac{\partial u}{\partial t} d x d t+\int_{\alpha}^{\beta}\left\{\left(a(x, 0)+\frac{\partial b(x, 0)}{\partial t}\right) \varphi^{2}+\left(\mathfrak{I}_{x} \mathcal{x}\right)^{2}\right\} d x \\
& +\int_{Q^{\tau}}\left(\frac{\partial a}{\partial t}+\frac{\partial^{2} b}{\partial t^{2}}\right) u^{2} d x d t-2 \int_{Q^{\tau}}\left(\frac{\partial a}{\partial x}+\frac{\partial^{2} b}{\partial x \partial t}\right) u \mathfrak{I}_{x} \frac{\partial u}{\partial t} d x d t \\
& -2 \int_{Q^{\tau}} \frac{\partial b}{\partial x} \frac{\partial u}{\partial t} \mathfrak{I}_{x} \frac{\partial u}{\partial t} d x d t-2 \int_{Q^{\tau}} \mathfrak{I}_{x}(c u) \mathfrak{I}_{x} \frac{\partial u}{\partial t} d x d t .
\end{aligned}
$$


Estimating the first and the three last integrals on the right-hand side of (2.7), by applying elementary inequalities, we get

$$
\begin{aligned}
& 2 \int_{Q^{\tau}} \mathfrak{I}_{x} f \mathfrak{I}_{x} \frac{\partial u}{\partial t} d x d t \leq \int_{Q^{\tau}}\left(\mathfrak{I}_{x} f\right)^{2} d x d t+\int_{Q^{\tau}}\left(\mathfrak{I}_{x} \frac{\partial u}{\partial t}\right)^{2} d x d t, \\
& -2 \int_{Q^{\top}}\left(\frac{\partial a}{\partial x}+\frac{\partial^{2} b}{\partial x \partial t}\right) u \mathfrak{I}_{x} \frac{\partial u}{\partial t} d x d t \leq 2 \int_{Q^{\top}}\left\{\left(\frac{\partial a}{\partial x}\right)^{2}+\left(\frac{\partial^{2} b}{\partial x \partial t}\right)^{2}\right\} u^{2} d x d t \\
& +\int_{Q^{\top}}\left(\mathfrak{J}_{x} \frac{\partial u}{\partial t}\right)^{2} d x d t \\
& -2 \int_{Q^{T}} \frac{\partial b}{\partial x} \frac{\partial u}{\partial t} \mathfrak{J}_{x} \frac{\partial u}{\partial t} d x d t \leq c_{4} \int_{Q^{T}}\left(\frac{\partial u}{\partial t}\right)^{2} d x d t \\
& +\frac{1}{c_{4}} \int_{Q^{\tau}}\left(\frac{\partial b}{\partial x}\right)^{2}\left(\mathfrak{J}_{x} \frac{\partial u}{\partial t}\right)^{2} d x d t \\
& -2 \int_{Q^{\tau}} \mathfrak{J}_{x}(c u) \mathfrak{I}_{x} \frac{\partial u}{\partial t} d x d t \leq \frac{(\beta-\alpha)^{2}}{2} \int_{Q^{\tau}} c^{2} u^{2} d x d t+\int_{Q^{\tau}}\left(\mathfrak{I}_{x} \frac{\partial u}{\partial t}\right)^{2} d x d t .
\end{aligned}
$$

Therefore, by formulas (2.7), (2.8), and Assumption 1.1, we obtain

$$
\begin{aligned}
\int_{0}^{\tau} \| & \frac{\partial u(\cdot, t)}{\partial t}\left\|_{L^{2}(\alpha, \beta)}^{2} d t+\right\| u(\cdot, \tau)\left\|_{L^{2}(\alpha, \beta)}^{2}+\right\| \mathfrak{J}_{x} \frac{\partial u(\cdot, \tau)}{\partial t} \|_{L^{2}(\alpha, \beta)}^{2} \\
\leq & c_{14}\left\{\int_{0}^{\tau}\left\|\mathfrak{I}_{x} f(\cdot, t)\right\|_{L^{2}(\alpha, \beta)}^{2} d t+\|\varphi\|_{L^{2}(\alpha, \beta)}^{2}+\left\|\mathfrak{J}_{x} \mathcal{\varkappa}\right\|_{L^{2}(\alpha, \beta)}^{2}\right\} \\
& +c_{15} \int_{0}^{\tau}\left\{\|u(\cdot, t)\|_{L^{2}(\alpha, \beta)}^{2}+\left\|\mathfrak{J}_{x} \frac{\partial u(\cdot, t)}{\partial t}\right\|_{L^{2}(\alpha, \beta)}^{2}\right\} d t
\end{aligned}
$$

where

$$
\begin{aligned}
c_{14} & =\frac{\max \left(1, c_{1}+c_{6}\right)}{\min \left(c_{4}, c_{0}+c_{5}, 1\right)}, \\
c_{15} & =\frac{\max \left(c_{2}+c_{8}+c_{3}^{2}+c_{9}^{2}, 3+c_{7}^{2} / c_{4}\right)}{\min \left(c_{4}, c_{0}+c_{5}, 1\right)} .
\end{aligned}
$$

Eliminating the last integral on the right-hand side of inequality (2.9). To this end, using Gronwall's lemma, it follows that

$$
\begin{aligned}
\int_{0}^{\tau}\left\|\frac{\partial u(\cdot, t)}{\partial t}\right\|_{L^{2}(\alpha, \beta)}^{2} d t+\|u(\cdot, \tau)\|_{L^{2}(\alpha, \beta)}^{2}+\left\|\mathfrak{J}_{x} \frac{\partial u(\cdot, \tau)}{\partial t}\right\|_{L^{2}(\alpha, \beta)}^{2} \\
\quad \leq c_{16}\left\{\int_{0}^{T}\left\|\mathfrak{J}_{x} f(\cdot, t)\right\|_{L^{2}(\alpha, \beta)}^{2} d t+\|\varphi\|_{L^{2}(\alpha, \beta)}^{2}+\left\|\mathfrak{I}_{x} \mathcal{K}\right\|_{L^{2}(\alpha, \beta)}^{2}\right\}
\end{aligned}
$$

where $c_{16}=c_{14} \exp \left(c_{15} T\right)$. 
The right-hand side of (2.11) is independent of $\tau$; hence, replacing the left-hand side by the upper bound with respect to $\tau$. Thus inequality (2.4) holds, where $C=c_{16}^{1 / 2}$.

It follows from (2.4) that there is a bounded inverse $L^{-1}$ on the range $R(L)$ of $L$. However, since we have no information concerning $R(L)$ expect that $R(L) \subset F$, we must extend $L$ (construct its closure $\bar{L}$ ) so that (2.4) holds for the extension and its range is the whole space.

We first show that $L: B \rightarrow F$ with domain $D(L)$, has a closure, that is, the closure of the graph $G(L) \subset B \times F$ of $L$ is a graph $G(\bar{L})=\overline{G(L)}$ of a new linear operator $\bar{L}$, which we call the closure of $L$.

Proposition 2.2. The operator $L$ from $B$ into $F$ has a closure.

Proof. Suppose that $u_{n} \in D(L)$ is a sequence such that

$$
\begin{gathered}
u_{n} \stackrel{n \rightarrow \infty}{\longrightarrow} 0 \text { in } B, \\
L u_{n} \stackrel{n \rightarrow \infty}{\longrightarrow}(f, \varphi, \varkappa) \text { in } F,
\end{gathered}
$$

we must prove that $f \equiv 0, \varphi \equiv 0$, and $\varkappa \equiv 0$. Equation (2.12) implies that

$$
u_{n} \stackrel{n \rightarrow \infty}{\longrightarrow} 0 \text { in } \mathscr{D}^{\prime}(Q)
$$

By virtue of the continuity of derivation of $\mathscr{D}^{\prime}(Q)$ in $\mathscr{D}^{\prime}(Q)$, we have

$$
\mathscr{L} u_{n} \stackrel{n \rightarrow \infty}{\longrightarrow} 0 \text { in } \mathscr{D}^{\prime}(Q) \text {. }
$$

We see via (2.13) that

$$
\mathscr{L} u_{n} \stackrel{n \rightarrow \infty}{\longrightarrow} f \text { in } L^{2}(Q),
$$

then

$$
\mathscr{L} u_{n} \stackrel{n \rightarrow \infty}{\longrightarrow} f \text { in } \mathscr{D}^{\prime}(Q) \text {. }
$$

By virtue of the uniqueness of the limit in $\mathscr{D}^{\prime}(Q),(2.15)$ and (2.17) imply that $f \equiv 0$. On the other hand, from (2.13) we have

$$
\ell_{1} u_{n} \stackrel{n \rightarrow \infty}{\longrightarrow} \varphi \text { in } L^{2}(\alpha, \beta)
$$

We see via (2.12) and the obvious inequality

$$
\left\|\ell_{1} u_{n}\right\|_{L^{2}(\alpha, \beta)} \leq\left\|u_{n}\right\|_{B}, \quad \forall n,
$$


that

$$
\ell_{1} u_{n} \stackrel{n \rightarrow \infty}{\longrightarrow} 0 \text { in } L^{2}(\alpha, \beta) .
$$

By virtue of (2.18), (2.20), and the uniqueness of the limit in $L^{2}(\alpha, \beta)$, we conclude that $\varphi \equiv 0$. The reasoning is similar for proving that $\mathcal{x} \equiv 0$.

DEFINITION 2.3. A solution of the equation

$$
\bar{L} u=(f, \varphi, \varkappa)
$$

is called a strong solution of problem (1.9), (1.10), (1.11), and (1.12).

Since points of the graph of $\bar{L}$ are limits of sequences of points of the graph of $L$, we extend (2.4) to apply to strong solutions by taking the limits.

COROLlary 2.4. Under the conditions of Theorem 2.1, there is a constant $C>0$ independent of $u$ such that

$$
\|u\|_{B} \leq C\|\bar{L} u\|_{F}, \quad \forall u \in D(\bar{L})
$$

Corollary 2.4 asserts that, if a strong solution exists, it is unique and depends continuously on $(f, \varphi, \varkappa)$, if $u$ is considered in the topology of $B$ and $(f, \varphi, \varkappa)$ is considered in the topology of $F$.

COROLLARY 2.5. The range $R(\bar{L})$ of the operator $\bar{L}$ equals to the closure $\overline{R(L)}$ of $R(L)$.

Proof. It follows from the definition of $\bar{L}$ that $R(\bar{L}) \subseteq \overline{R(L)}$. It remains to prove the opposite inclusion. Suppose that $w \in \overline{R(L)}$, then there exists a sequence $\left\{w_{n}\right\}_{n=1}^{\infty}$ of elements in $R(L)$ such that $\lim _{n \rightarrow \infty} w_{n}=w$. Consequently, there exists a corresponding sequence $u_{n} \in D(L)$ such that $L u_{n}=w_{n}$.

According to Theorem 2.1, we have

$$
\left\|u_{m}-u_{n}\right\|_{B} \leq C\left\|L u_{m}-L u_{n}\right\|_{F}
$$

when $n$ and $m \rightarrow \infty$. Thus $\left\{u_{n}\right\}$ is a fundamental sequence in $B$ which converges to an element $u \in B$ and $\bar{L} u=w$, then $w \in R(\bar{L})$. This proves Corollary 2.5.

Corollary 2.5 states that, to prove that problem (1.9), (1.10), (1.11), and (1.12) has a strong solution for arbitrary $(f, \varphi, \varkappa) \in F$, it is sufficient to show that $\overline{R(L)}=F$.

\section{Solvability of the problem}

THEOREM 3.1. Let Assumptions 1.1 and 1.2 be fulfilled. Then for any $\mathfrak{J}_{x} f \in L^{2}(Q)$, $\varphi \in L^{2}(\alpha, \beta)$, and $\mathfrak{J}_{x} \varkappa \in L^{2}(\alpha, \beta)$, problem (1.9), (1.10), (1.11), and (1.12) admits a unique strong solution $u=\bar{L}^{-1}(f, \varphi, \varkappa)=\overline{L^{-1}}(f, \varphi, \varkappa)$. 
Proof. First, we prove that $R(L)$ is dense in $F$ for the special case in which $L$ is reduced to $L_{0}=\left(\mathscr{L}_{0}, \ell_{1}, \ell_{2}\right)$ with domain $D_{0}\left(L_{0}\right)=D_{0}(L)$, where $\mathscr{L}_{0}$ is the principal part of $\mathscr{L}$, that is,

$$
\mathscr{L}_{0} u=\frac{\partial^{2} u}{\partial t^{2}}-\frac{\partial}{\partial x}\left(a(x, t) \frac{\partial u}{\partial x}\right)-\frac{\partial^{2}}{\partial t \partial x}\left(b(x, t) \frac{\partial u}{\partial x}\right),
$$

and $D_{0}(L)=\left\{u / u \in D(L): \ell_{1} u=0\right.$ and $\left.\ell_{2} u=0\right\}$.

Proposition 3.2. Under the conditions of Theorem 3.1. If

$$
\int_{Q} \mathfrak{I}_{x} \mathscr{L}_{0} u \cdot \mathfrak{I}_{x} \omega d x d t=0
$$

for $\mathfrak{J}_{x} \omega \in L^{2}(Q)$ and for all $u \in D_{0}(L)$, then $\omega$ vanishes almost everywhere in $Q$.

Proof of Proposition 3.2. Construct the function $\mathfrak{J}_{x} \omega$. Using the fact that relation (3.2) holds for any function $u \in D_{0}(L)$, we can express $\mathfrak{I}_{x} u$ in a special form. Let

$$
\mathfrak{J}_{x} u= \begin{cases}0, & 0 \leq t \leq s, \\ \int_{s}^{t}(t-\tau) \frac{\partial^{2}\left(\mathfrak{J}_{x} u\right)}{\partial \tau^{2}} d \tau, & s \leq t \leq T,\end{cases}
$$

and let $\mathfrak{J}_{x}\left(\partial^{2} u / \partial t^{2}\right)$ be a solution of the equation

$$
a(\sigma, t) \mathfrak{I}_{x} \frac{\partial^{2} u}{\partial t^{2}}=\mathfrak{I}_{t}^{*}\left(\mathfrak{I}_{x} \omega\right)=\int_{t}^{T} \mathfrak{I}_{x} \omega d \tau,
$$

where $\sigma$ is a fixed number in $[\alpha, \beta]$. We now have

$$
\mathfrak{I}_{x} \omega=\mathfrak{I}_{t}^{*-1}\left(a(\sigma, t) \mathfrak{I}_{x} \frac{\partial^{2} u}{\partial t^{2}}\right)=-\frac{\partial}{\partial t}\left(a(\sigma, t) \mathfrak{I}_{x} \frac{\partial^{2} u}{\partial t^{2}}\right) .
$$

Relations (3.3) and (3.4) imply that $u$ is in $D_{s}(L)$, where $D_{s}(L)$ is the set of functions $D(L)$ such that $u$ and $\partial u / \partial t$ vanish in the neighborhood of $t \leq s$. If we put $s=0$, then $u$ is in $D_{0}(L)$.

LEMMA 3.3. Under the conditions of Proposition 3.2, the function $\mathfrak{J}_{x} u$, defined by (3.3) and (3.4), has derivatives with respect to $t$ up to third-order inclusive belonging to the space $L^{2}\left(Q_{s}\right)$, where $Q_{s}=(\alpha, \beta) \times(s, T)$.

The proof of Lemma 3.3 is similar to that of [4, Lemma 1].

Substituting (3.1) and (3.5) into (3.2), we have

$$
\begin{aligned}
-\int_{Q_{s}} \mathfrak{I}_{x} & \frac{\partial^{2} u}{\partial t^{2}} \frac{\partial}{\partial t}\left(a(\sigma, t) \mathfrak{I}_{x} \frac{\partial^{2} u}{\partial t^{2}}\right) d x d t \\
& +\int_{Q_{s}} a \frac{\partial u}{\partial x} \frac{\partial}{\partial t}\left(a(\sigma, t) \mathfrak{I}_{x} \frac{\partial^{2} u}{\partial t^{2}}\right) d x d t \\
& +\int_{Q_{s}} \frac{\partial}{\partial t}\left(b \frac{\partial u}{\partial x}\right) \frac{\partial}{\partial t}\left(a(\sigma, t) \mathfrak{I}_{x} \frac{\partial^{2} u}{\partial t^{2}}\right) d x d t=0
\end{aligned}
$$


Integrating by parts each term of the above equality, we obtain

$$
\begin{aligned}
&-\int_{Q_{s}} \mathfrak{I}_{x} \frac{\partial^{2} u}{\partial t^{2}} \frac{\partial}{\partial t}\left(a(\sigma, t) \mathfrak{I}_{x} \frac{\partial^{2} u}{\partial t^{2}}\right) d x d t \\
&= \frac{1}{2} \int_{\alpha}^{\beta} a(\sigma, s)\left(\mathfrak{J}_{x} \frac{\partial^{2} u(\xi, s)}{\partial t^{2}}\right)^{2} d x-\frac{1}{2} \int_{Q_{s}} a^{\prime}(\sigma, t)\left(\mathfrak{I}_{x} \frac{\partial^{2} u}{\partial t^{2}}\right)^{2} d x d t \\
& \int_{Q_{s}} a \frac{\partial u}{\partial x} \frac{\partial}{\partial t}\left(a(\sigma, t) \mathfrak{J}_{x} \frac{\partial^{2} u}{\partial t^{2}}\right) d x d t \\
&= \int_{Q_{s}}\left(a \frac{\partial u}{\partial t}+\frac{\partial a}{\partial t} u\right) a(\sigma, t) \frac{\partial^{2} u}{\partial t^{2}} d x d t+\int_{Q_{s}}\left(\frac{\partial a}{\partial x} \frac{\partial u}{\partial t}+\frac{\partial^{2} a}{\partial t \partial x} u\right) a(\sigma, t) \mathfrak{I}_{x} \frac{\partial^{2} u}{\partial t^{2}} d x d t \\
& \int_{Q_{s}} \frac{\partial}{\partial t}\left(b \frac{\partial u}{\partial x}\right) \frac{\partial}{\partial t}\left(a(\sigma, t) \mathfrak{I}_{x} \frac{\partial^{2} u}{\partial t^{2}}\right) d x d t \\
&=\int_{Q_{s}} b a(\sigma, t)\left(\frac{\partial^{2} u}{\partial t^{2}}\right)^{2} d x d t+\int_{\alpha}^{\beta} \frac{\partial b}{\partial t} a(\sigma, T)\left(\frac{\partial u(x, T)}{\partial t}\right)^{2} d x \\
& \quad-\frac{1}{2} \int_{Q_{s}} \frac{\partial^{2} b}{\partial x^{2}} a(\sigma, t)\left(\mathfrak{I}_{x} \frac{\partial^{2} u}{\partial t^{2}}\right)^{2} d x d t-\int_{Q_{s}}\left(\frac{\partial^{2} b}{\partial t^{2}} a(\sigma, t)+\frac{\partial b}{\partial t} a^{\prime}(\sigma, t)\right)\left(\frac{\partial u}{\partial t}\right)^{2} d x d t \\
& \quad+2 \int_{Q_{s}} \frac{\partial^{2} b}{\partial x \partial t} a(\sigma, t) \frac{\partial u}{\partial t} \mathfrak{I}_{x} \frac{\partial^{2} u}{\partial t^{2}} d x d t+\int_{Q_{s}} \frac{\partial^{2} b}{\partial t^{2}} a(\sigma, t) u \frac{\partial^{2} u}{\partial t^{2}} d x d t \\
&+\int_{Q_{s}} \frac{\partial^{3} b}{\partial x \partial t^{2}} a(\sigma, t) u \mathfrak{J}_{x} \frac{\partial^{2} u}{\partial t^{2}} d x d t .
\end{aligned}
$$

Substituting (3.7) into (3.6) yields

$$
\begin{aligned}
\int_{\alpha}^{\beta}\{ & \left.\frac{a(\sigma, s)}{2}\left(\mathfrak{J}_{x} \frac{\partial^{2} u(\xi, s)}{\partial t^{2}}\right)^{2}+\frac{\partial b}{\partial t} a(\sigma, T)\left(\frac{\partial u(x, T)}{\partial t}\right)^{2}\right\} d x \\
& +\int_{Q_{s}} b a(\sigma, t)\left(\frac{\partial^{2} u}{\partial t^{2}}\right)^{2} d x d t \\
= & \frac{1}{2} \int_{Q_{s}}\left(a^{\prime}(\sigma, t)+\frac{\partial^{2} b}{\partial x^{2}} a(\sigma, t)\right)\left(\mathfrak{J}_{x} \frac{\partial^{2} u}{\partial t^{2}}\right)^{2} d x d t \\
& +\int_{Q_{s}}\left(\frac{\partial^{2} b}{\partial t^{2}} a(\sigma, t)+\frac{\partial b}{\partial t} a^{\prime}(\sigma, t)\right)\left(\frac{\partial u}{\partial t}\right)^{2} d x d t \\
& -\int_{Q_{s}}\left\{a \frac{\partial u}{\partial t}+\left(\frac{\partial a}{\partial t}+\frac{\partial^{2} b}{\partial t^{2}}\right) u\right\} a(\sigma, t) \frac{\partial^{2} u}{\partial t^{2}} d x d t \\
& -\int_{Q_{s}}\left\{\left(\frac{\partial a}{\partial x}+2 \frac{\partial^{2} b}{\partial x \partial t}\right) \frac{\partial u}{\partial t}+\left(\frac{\partial^{2} a}{\partial t \partial x}+\frac{\partial^{3} b}{\partial x \partial t^{2}}\right) u\right\} a(\sigma, t) \mathfrak{I}_{x} \frac{\partial^{2} u}{\partial t^{2}} d x d t
\end{aligned}
$$


By virtue of Assumptions 1.1 and 1.2, we obtain

$$
\begin{aligned}
& \frac{c_{0}}{2}\left\|\mathfrak{J}_{x} \frac{\partial^{2} u(\cdot, s)}{\partial t^{2}}\right\|_{L^{2}(\alpha, \beta)}^{2}+c_{0} c_{5}\left\|\frac{\partial u(\cdot, T)}{\partial t}\right\|_{L^{2}(\alpha, \beta)}^{2} \\
& \leq \frac{1}{2}\left\{c_{2}+c_{1} c_{12}+\frac{c_{1}^{2}}{2}\right\} \int_{s}^{T}\left\|\mathfrak{J}_{x} \frac{\partial^{2} u(\cdot, t)}{\partial t^{2}}\right\|_{L^{2}(\alpha, \beta)}^{2} d t \\
&+\left\{c_{1} c_{8}+c_{2} c_{6}+\frac{c_{4}^{2}}{2 c_{0} c_{4}}+c_{3}^{2}+4 c_{9}^{2}\right\} \int_{s}^{T}\left\|\frac{\partial u(\cdot, t)}{\partial t}\right\|_{L^{2}(\alpha, \beta)}^{2} d t \\
&+\left\{\frac{c_{1}^{2}\left(c_{2}^{2}+c_{8}^{2}\right)}{2 c_{0} c_{4}}+c_{11}^{2}+c_{13}^{2}\right\} \int_{s}^{T}\|u(\cdot, t)\|_{L^{2}(\alpha, \beta)}^{2} d t .
\end{aligned}
$$

Using the Friedrichs inequality for the norm of $u$ obtained from the norm of $\partial u / \partial t$. This yields

$$
\begin{aligned}
& \left\|\mathfrak{J}_{x} \frac{\partial^{2} u(\cdot, s)}{\partial t^{2}}\right\|_{L^{2}(\alpha, \beta)}^{2}+\left\|\frac{\partial u(\cdot, T)}{\partial t}\right\|_{L^{2}(\alpha, \beta)}^{2} \\
& \quad \leq c_{17} \int_{S}^{T}\left\{\left\|\mathfrak{J}_{x} \frac{\partial^{2} u(\cdot, t)}{\partial t^{2}}\right\|_{L^{2}(\alpha, \beta)}^{2}+\left\|\frac{\partial u(\cdot, t)}{\partial t}\right\|_{L^{2}(\alpha, \beta)}^{2}\right\} d t,
\end{aligned}
$$

where

$$
\begin{aligned}
c_{17}= & \left.\max \left(\frac{c_{2}+c_{1} c_{12}}{2}+\frac{c_{1}^{2}}{4}, c_{1} c_{8}+c_{2} c_{6}+\frac{c_{4}^{2}}{2 c_{0} c_{4}}+c_{3}^{2}+4 c_{9}^{2}+\gamma\left(\frac{c_{1}^{2}\left(c_{2}^{2}+c_{8}^{2}\right)}{2 c_{0} c_{4}}+c_{11}^{2}+c_{13}^{2}\right)\right)\right] \\
& \times\left[\min \left(\frac{c_{0}^{2}}{2}, c_{0} c_{5}\right)\right]^{-1}
\end{aligned}
$$

and $\gamma$ is the constant of the Friedricks inequality.

Inequality (3.10) is basic in our proof. In order to use it, we introduce a new function $z$ defined by the formula

$$
z(x, t)=\mathfrak{J}_{t}^{*} \frac{\partial^{2} u}{\partial \tau^{2}}=\int_{t}^{T} \frac{\partial^{2} u}{\partial \tau^{2}} d \tau
$$

Then, $\partial u(x, t) / \partial t=z(x, s)-z(x, t), \partial u(x, T) / \partial t=z(x, s)$, and we have

$$
\int_{S}^{T}\left\|\frac{\partial u(\cdot, t)}{\partial t}\right\|_{L^{2}(\alpha, \beta)}^{2} d t \leq 2\left\{\int_{S}^{T}\|z(\cdot, t)\|_{L^{2}(\alpha, \beta)}^{2} d t+(T-s)\|z(\cdot, s)\|_{L^{2}(\alpha, \beta)}^{2}\right\} .
$$

Consequently, (3.10) becomes

$$
\begin{aligned}
& \left\|\mathfrak{J}_{x} \frac{\partial^{2} u(\cdot, s)}{\partial t^{2}}\right\|_{L^{2}(\alpha, \beta)}^{2}+\left(1-2 c_{17}(T-s)\right)\|z(\cdot, s)\|_{L^{2}(\alpha, \beta)}^{2} \\
& \quad \leq 2 c_{17} \int_{s}^{T}\left\{\left\|\mathfrak{I}_{x} \frac{\partial^{2} u(\cdot, t)}{\partial t^{2}}\right\|_{L^{2}(\alpha, \beta)}^{2}+\|z(\cdot, t)\|_{L^{2}(\alpha, \beta)}^{2}\right\} d t .
\end{aligned}
$$


Hence, if $s_{0}>0$ satisfies $1-2 c_{17}(T-s)=1 / 2$, then inequality (3.14) implies

$$
\begin{aligned}
& \left\|\mathfrak{J}_{x} \frac{\partial^{2} u(\cdot, s)}{\partial t^{2}}\right\|_{L^{2}(\alpha, \beta)}^{2}+\|z(\cdot, s)\|_{L^{2}(\alpha, \beta)}^{2} \\
& \quad \leq 4 c_{17} \int_{s}^{T}\left\{\left\|\mathfrak{I}_{x} \frac{\partial^{2} u(\cdot, t)}{\partial t^{2}}\right\|_{L^{2}(\alpha, \beta)}^{2}+\|z(\cdot, t)\|_{L^{2}(\alpha, \beta)}^{2}\right\} d t
\end{aligned}
$$

for all $s \in\left[T-s_{0}, T\right]$. We denote the integral on the right-hand side of (3.15) by $y(s)$. Hence, we obtain

$$
-\frac{d y(s)}{d s} \leq 4 c_{17} y(s)
$$

and, consequently,

$$
-\frac{d}{d s}\left(y(s) \exp \left(4 c_{17} s\right)\right) \leq 0 .
$$

It follows from (3.17) that $y(s)=0$, and thus $\mathfrak{I}_{x} \omega \equiv 0$ almost everywhere in $Q_{T-s_{0}}$. Proceeding in this way step by step along a rectangle of side $s_{0}$, we prove that $\mathfrak{I}_{x} \omega \equiv 0$, and thus $\omega \equiv 0$ almost everywhere in $Q$.

Now, we will prove Theorem 3.1. For this end in view, it is sufficient to prove that the range $R(L)$ of $L$ is dense in $F$. Suppose that, for some $W=\left(\omega, \omega_{1}, \omega_{2}\right) \in F$ be orthogonal to $R\left(L_{0}\right)$, so that

$$
\int_{Q} \mathfrak{J}_{x} \mathscr{L}_{0} u \cdot \mathfrak{J}_{x} \omega d x d t+\int_{\alpha}^{\beta} \ell_{1} u \omega_{1} d x+\int_{\alpha}^{\beta} \mathfrak{I}_{x} \ell_{2} u \mathfrak{I}_{x} \omega_{2} d x=0 .
$$

We must prove that $W \equiv 0$. Putting $u \in D_{0}(L)$ in (3.18), we obtain

$$
\int_{Q} \mathfrak{J}_{x} \mathscr{L}_{0} u \cdot \mathfrak{J}_{x} \omega d x d t=0, \quad u \in D_{0}(L) .
$$

Hence Proposition 3.2 implies that $\omega \equiv 0$. Thus (3.18) takes the form

$$
\int_{\alpha}^{\beta} \ell_{1} u \omega_{1} d x+\int_{\alpha}^{\beta} \mathfrak{I}_{x} \ell_{2} u \mathfrak{I}_{x} \omega_{2} d x=0 .
$$

Since $\ell_{1}$ and $\ell_{2}$ are independent and the sets of the operators $\ell_{1}$ and $\ell_{2}$ are everywhere dense in $L^{2}(\alpha, \beta)$ and the space with the norm $\left(\int_{\alpha}^{\beta}\left(\mathfrak{J}_{x} \omega_{2}\right)^{2} d x\right)^{1 / 2}$, respectively, the above relation implies that $\omega_{1} \equiv 0$ and $\omega_{2} \equiv 0$. Hence $W \equiv 0$, and thus $\overline{R\left(L_{0}\right)}=F$.

Now consider the general case. If we use the fact that $R\left(L_{0}\right)$ is dense in $F$ and that $L-L_{0}=\left(\mathscr{L}-\mathscr{L}_{0}, \ell_{1}, \ell_{2}\right)$ maps continuously $B$ into $F$, we conclude that we can prove that $R(L)$ is dense in $F$ by means of the method of continuation along the parameter. We will not describe the application of this method because it is analogous to the method used in [3].

ACKNOWLEDGMent. This work was supported by "Le Centre Universitaire Larbi Ben M'hidi-Oum El Baouagui." 


\section{REFERENCES}

[1] G. W. Batten Jr., Second-order correct boundary conditions for the numerical solution of the mixed boundary problem for parabolic equations, Math. Comp. 17 (1963), 405413.

[2] N. E. Benouar and N. I. Yurchuk, A mixed problem with an integral condition for parabolic equations with a Bessel operator, Differentsial'nye Uravneniya 27 (1991), no. 12, 2094-2098.

[3] A. Bouziani, Mixed problem for certain non-classical equations containing a small parameter, Acad. Roy. Belg. Bull. Cl. Sci. (6) 5 (1994), no. 7-12, 389-400.

[4] _ Mixed problem with boundary integral conditions for a certain parabolic equation, J. Appl. Math. Stochastic Anal. 9 (1996), no. 3, 323-330.

[5] __ Solution forte d'un problème mixte avec une condition non locale pour une classe d'équations hyperboliques, Acad. Roy. Belg. Bull. Cl. Sci. (6) 8 (1997), no. 1-6, 53-70 (French).

[6] Strong solution for a mixed problem with nonlocal condition for certain pluriparabolic equations, Hiroshima Math. J. 27 (1997), no. 3, 373-390.

[7] __ On a third order parabolic equation with a nonlocal boundary condition, J. Appl. Math. Stochastic Anal. 13 (2000), no. 2, 181-195.

[8] J. R. Cannon, The solution of the heat equation subject to the specification of energy, Quart. Appl. Math. 21 (1963), 155-160.

[9] J. R. Cannon, S. P. Esteva, and J. van der Hoek, A Galerkin procedure for the diffusion equation subject to the specification of mass, SIAM J. Numer. Anal. 24 (1987), no. 3, 499-515.

[10] J. R. Cannon and J. van der Hoek, The existence of and a continuous dependence result for the solution of the heat equation subject to the specification of energy, Boll. Un. Mat. Ital. Suppl. (1981), no. 1, 253-282.

[11]___ An implicit finite difference scheme for the diffusion equation subject to the specification of mass in a portion of the domain, Numerical Solutions of Partial Differential Equations (Parkville, 1981) (J. Noye, ed.), North-Holland Publishing, Amsterdam, 1982 , pp. 527-539.

[12] N. I. Ionkin, The solution of a certain boundary value problem of the theory of heat conduction with a nonclassical boundary condition, Differencial'nye Uravnenija 13 (1977), no. 2, 294-304.

[13] Y Lin, Parabolic partial differential equations subject to nonlocal boundary conditions, Ph.D. thesis, Washington State University, Pullman, 1988.

[14] S. Mesloub and A. Bouziani, On a class of singular hyperbolic equation with a weighted integral condition, Int. J. Math. Math. Sci. 22 (1999), no. 3, 511-519.

[15] P. Shi, Weak solution to an evolution problem with a nonlocal constraint, SIAM J. Math. Anal. 24 (1993), no. 1, 46-58.

[16] N. I. Yurchuk, A mixed problem with an integral condition for some parabolic equations, Differentsial'nye Uravneniya 22 (1986), no. 12, 2117-2126.

Abdelfatah Bouziani: Département de Mathématiques, Centre Universitaire LaRbi Ben M'Hidi-OUm El BAOUAGUi, 04000, Algeria

Current address: MATHEMATICAL Division, The AbDus SALAm INTERNATIONAL CENTRE FOR

TheOretical Physics (ICTP), STRADA Costiera 11, 34100 Trieste, ITALY

E-mail address: bouziani@ictp.trieste.it 


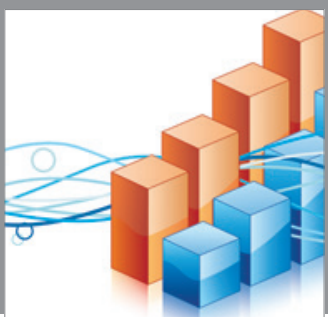

Advances in

Operations Research

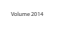

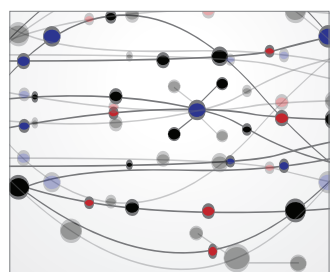

\section{The Scientific} World Journal
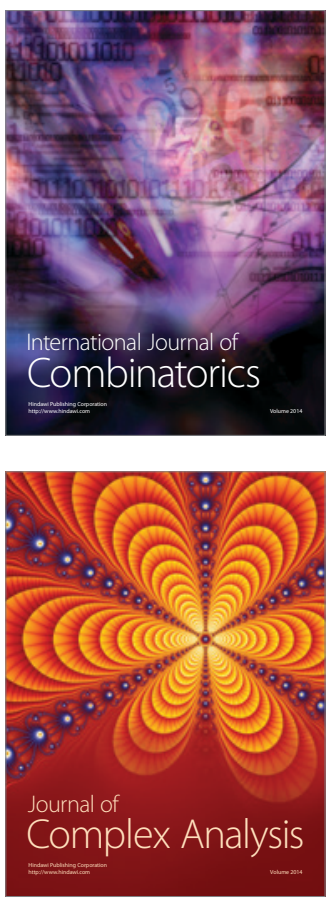

International Journal of

Mathematics and

Mathematical

Sciences
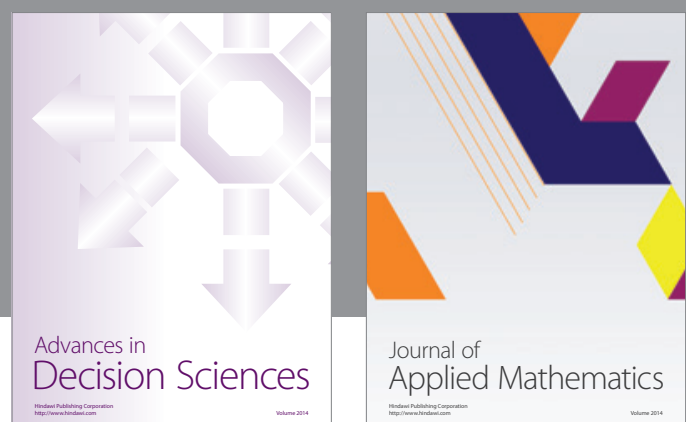

Journal of

Applied Mathematics
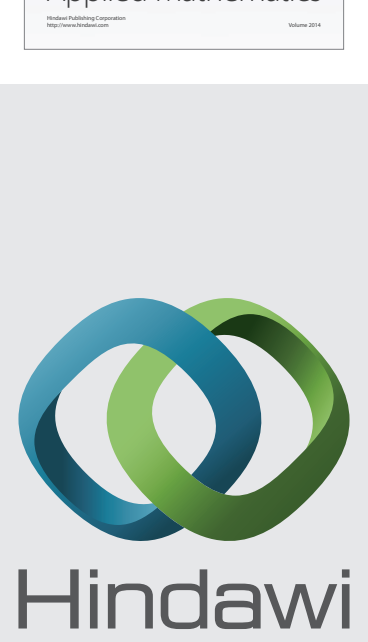

Submit your manuscripts at http://www.hindawi.com
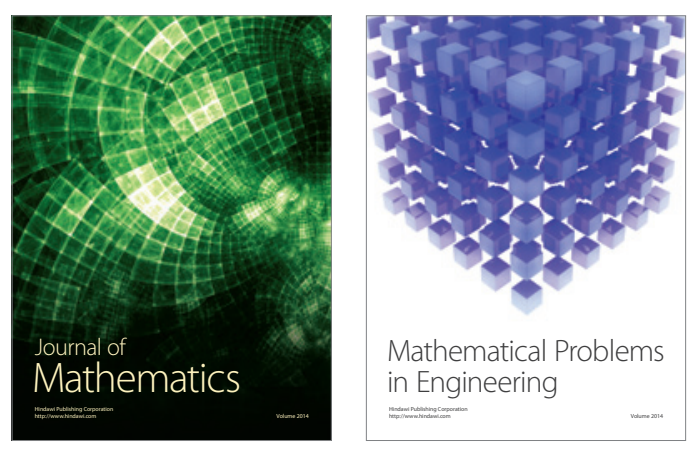

Mathematical Problems in Engineering
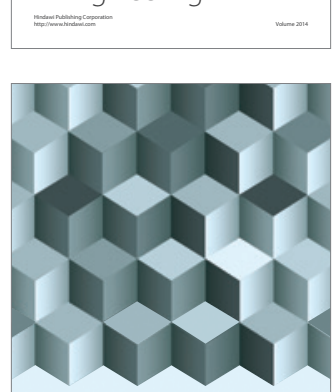

Journal of

Function Spaces
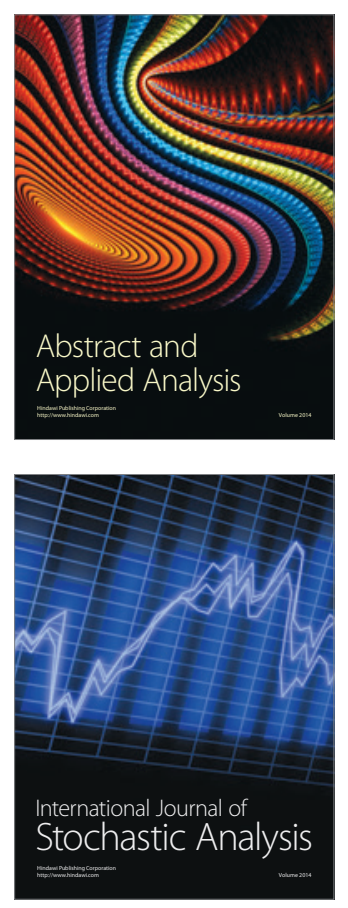

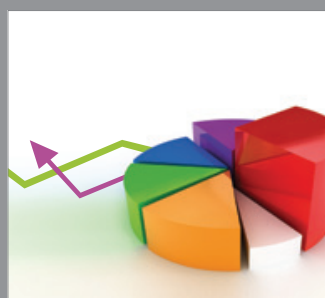

ournal of

Probability and Statistics

Promensencen
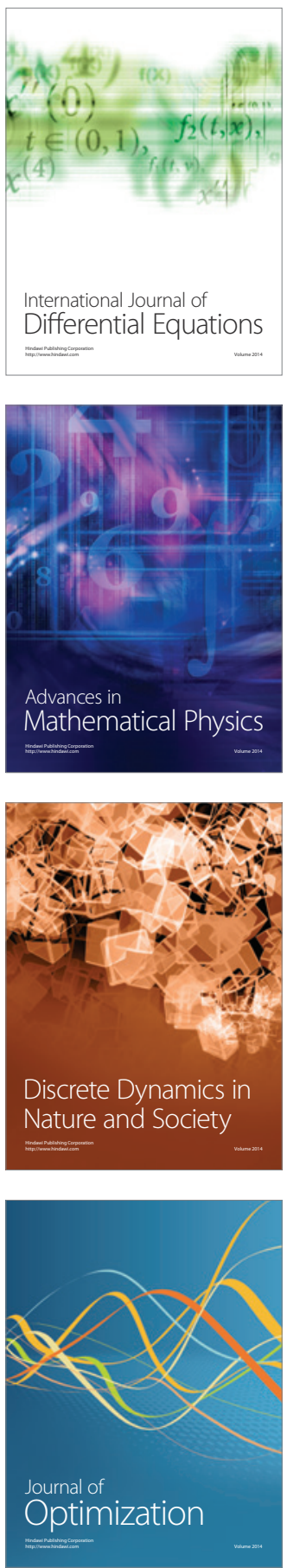